Cahiers $d u$ MONDE RUSSE

\section{Cahiers du monde russe}

Russie - Empire russe - Union soviétique et États indépendants

40/1-2| 1999

Archives et nouvelles sources de l'histoire soviétique, une réévaluation

\title{
Les fonds personnels des dirigeants soviétiques
}

Histoire de leur formation et état actuel

\section{Lidija Kosheleva et Larisa Rogovaja}

\section{(2) OpenEdition}

\section{Journals}

Édition électronique

URL : http://journals.openedition.org/monderusse/4

DOI : $10.4000 /$ monderusse.4

ISSN : 1777-5388

Éditeur

Éditions de l'EHESS

Édition imprimée

Date de publication : 1 janvier 1999

Pagination : $91-100$

ISBN : 2-7132-1314-2

ISSN : $1252-6576$

Référence électronique

Lidija Kosheleva et Larisa Rogovaja, «Les fonds personnels des dirigeants soviétiques », Cahiers du monde russe [En ligne], 40/1-2 | 1999, mis en ligne le 15 janvier 2007, Consulté le 20 avril 2019. URL http://journals.openedition.org/monderusse/4 ; DOI : 10.4000/monderusse.4 


\title{
LES FONDS PERSONNELS DES DIRIGEANTS SOVIÉTIQUES
}

\author{
Histoire de leur formation et état actuel
}

Plus DE 500 FONDS D'ARCHIVES, dont la moitié proviennent de collections et de fonds d'origine privée, ont été rassemblés au Centre de Conservation et d'Étude des Documents sur l'Histoire Contemporaine de Russie (Rossijskij Centr Hranenija i Izučenija Dokumentov Noveijšej Istorii - RCHIDNI), créé le 12 octobre 1991 à partir des anciennes Archives Centrales du Parti Communiste (Central'nyj Partijnyj Arhiv - CPA). Ce chiffre suffit à montrer l'importance des fonds personnels des dirigeants du parti et de l'État en tant que source historique.

L'histoire de la formation des fonds personnels conservés au RCHIDNI pourrait faire l'objet d'une recherche spécialisée approfondie. L'objectif de cet article est d'exposer brièvement les principes de la constitution de ces fonds personnels, notamment de ceux qui y sont entrés au cours des dernières années, et qui venaient des Archives du Président de la Fédération de Russie (AP RF), ex-archives du Politbjuro du Comité Central du parti (CK VKP(b)-KPSS). Il est évident que ces documents, qui avaient été conservés dans le saint des saints, éveillent le plus grand intérêt chez les chercheurs. Ce sont avant tout les fonds de V. M. Molotov, L. M. Kaganovič, K. E. Vorošilov, A. I. Rykov, L. B. Kamenev, G. E. Zinov'ev, G. V. Čičerin, A. A.Ždanov, M. M. Litvinov, N. I. Ežov et d'autres.

Il faut remarquer que, lors de l'entrée des documents des Archives du Président, le RCHIDNI conservait déjà les fonds de nombreux dirigeants du parti et du gouvernement, arrivés à des moments différents et provenant de sources variées. Les fonds les plus complets ont été obtenus après le transfert, depuis les archives du parti, de documents de dirigeants décédés (G. K. Ordžonikidze, A. A. Ždanov, S. M. Kirov). Un certain nombre de fonds personnels étaient en fait des collections de documents occasionnels découverts dans le CPA et dans d'autres archives (I. V. Stalin, V. M. Molotov). L'étude scientifique de ces documents a été entreprise immédiatement par le CPA avec pour principe de base la description du document : 
détermination de la date, de l'auteur et de ses correspondants, long commentaire du contenu de chaque document au début de l'inventaire (opis'). Ainsi ont été décrits les fonds de G. K. Ordžonikidze, A. A. Ždanov, S. M. Kirov, V. M. Molotov, A. A. Andreev, K. E. Vorošilov, E. M. Jaroslavskij et d'autres.

Les documents des fonds personnels issus des Archives du Président permettent d'observer comment, à partir de quelles sources, ces fonds ont été constitués, ce qui a une grande importance pour leur étude et pour la recherche des documents indispensables. D'après leur provenance, on peut diviser les fonds personnels des anciennes archives du Politbjuro en trois groupes :

1. Les documents transmis à ces archives par décision du Comité Central (CC) du parti à l'occasion de la mort d'un dirigeant, ce qui donnait lieu à la création de commissions du CC pour la réception des archives.

Ainsi entrèrent dans les archives du Politbjuro les fonds de G. K. Ordžonikidze (par décision du Politbjuro du 21 février 1937, la commission - composée de A. I. Mikojan (président), L. Z. Mehlis, A. D. Semuškin et L. P. Berija - fut chargée de « réceptionner toutes les archives d'Ordžonikidze ainsi que sa correspondance avec des camarades, d'y mettre de l'ordre et de faire un rapport au Comité Central du parti $»^{1}$ ), les fonds de M. M. Litvinov (en 1952), de E. M. Jaroslavskij (en 1943), et de A. A. ̌̌danov (en 1948).

2. Les documents transmis par décision du Comité Central, à l'occasion de la destitution ou du départ à la retraite d'un dirigeant. Ainsi furent recueillis dans les archives du Politbjuro les documents de A. Ja. Vyšinskij (en 1954), A. I. Mikojan (en 1966), N. I. Ežov (en 1938), G. M. Malenkov (en 1957), L. M. Kaganovič (en 1957), N. M. Ÿvernik (en 1969).

3. Les documents du Commissariat du Peuple à l'Intérieur (Narodnyj Komissariat Vnutrennih Del - NKVD). Les documents de N. I. Buharin, G. E. Zinov'ev, L. B. Kamenev, L. D. Trockij, A. I. Rykov furent transmis aux archives du Politbjuro en 1937, ceux de K. B. Radek en 1939. On ne connaît pas la date exacte d'entrée dans les archives du Politbjuro des documents de N. M. Goloded, S. A. Lozovskij, M. D. Orahelašvili, A. S. Svanidze, I. F. Kodackij, Ÿ. Z. Elijava, mais les limites chronologiques de ces documents et leur nature (pour l'essentiel des lettres, des déclarations, de la correspondance) laissent à penser qu'ils furent également transmis par le NKVD.

L'histoire de la constitution du fonds de N. I. Ežov présente un intérêt particulier. Le 5 décembre 1938, le Comité Central du parti et le Conseil des Commissaires du Peuple (Sovet Narodnyh Komissarov - SNK) ont pris une résolution sur le dépôt des dossiers concernant le NKVD, conformément à laquelle Ežov s'engageait à remettre tous ses dossiers (dela) au nouveau commissaire du peuple aux Affaires intérieures, L. P. Berija. La remise devait se faire en présence du secrétaire du Comité Central, A. A. Andreev, et du directeur de la Section des Organes Dirigeants du Parti (Otdel Rukovodjaščih Partijnyh Organov - ORPO),

1. RCHIDNI,f. 17, op. 3, d. $984,1.5$. 
G. M. Malenkov². En mars 1939, une autre commission composée de G. M. Malenkov, du responsable du Secteur Spécial (Osobyj Sektor) du Comité Central, A. N. Poskrebyšev, et du chef du service administratif du Comité Central, D. V. Krupin, fut chargée de réceptionner tous les dossiers concernant le secrétariat du Comité Central de l'ancien secrétaire, Ežov. Le 17 octobre 1940, Krupin remit aux archives du Secteur Spécial du CC (c'est-à-dire aux archives du Politbjuro) les documents de l'ex-secrétaire du Comité Central, Ežov. Dans le même temps, une partie des documents fut transmise aux archives de l'Orgbjuro et du Secrétariat - aujourd'hui ces documents se trouvent en partie au RCHIDNI, opisi 120 et 121, fond 17 (Secrétariat technique du Comité Central, constitué de documents des secrétariats des secrétaires du CC).

Déposées au Secteur Spécial du CC en 1940, les archives de Ežov comprenaient les documents de L. D. Trockij, G. Ja. Sokol'nikov, G. V. Čičerin, A. S. Enukidze. On ne s'explique pas les raisons pour lesquelles ces papiers se sont retrouvés chez Ežov, mais on peut supposer que cela est lié à son activité de président de la Commission de Contrôle du Parti (Komissija Partijnogo Kontrolja - KPK) et surtout de commissaire aux Affaires intérieures, c'est-à-dire à l'organisation des répressions.

Avant 1956, presque tous les fonds d'archives du Politbjuro se trouvaient sous forme de liasses éparpillées, ce n'est qu'au cours des années 1956-1958, probablement en raison de la campagne de réhabilitation, après le $\mathrm{XX}^{\mathrm{e}}$ congrès, qu'un travail scientifique et technique fut entrepris sur les principales collections de documents du Secteur Spécial du CC (les archives du Politbjuro). Une fois ce travail accompli, les documents de Trockij, Sokol'nikov, Enukidze, Čičerin furent transférés du fonds Ežov vers des fonds indépendants (en tout 123 dossiers). Les 219 dossiers restants (pour l'essentiel des documents des années 1933 à 1938) ont été classés selon un ordre thématique et, à l'intérieur des dossiers, de façon chronologique. Une part importante des documents reflète l'activité de Ežov comme dirigeant de l'enquête dans l'assassinat de Kirov, comme organisateur actif des répressions contre les membres de l'ancienne opposition, mais également des grands procès politiques de 1935-1937 parmi lesquels «le bloc antisoviétique trotskiste de droite », «le centre antisoviétique parallèle trotskiste de droite », etc. Ežov témoigna de son expérience dans la lutte contre l'opposition et de sa conception de la lutte des classes dans la société et le parti, dans le manuscrit de son livre Ot frakcionnosti k otkrytoj kontrrevoljucii (De la fraction à la contre-révolution ouverte). Les matériaux de son manuscrit, quelques-unes de ses variantes, mais aussi un exemplaire transmis par Ežov à Stalin en 1935, sont conservés dans ce fonds. Il est indéniable que les corrections et les remarques de Stalin, et d'autres collaborateurs du CC, dont V. G. Knorin, L. Z. Mehlis, P. N. Pospelov, A. I. Steckij, M. F. Ÿkirjatov, E. M. Jaroslavskij, sont d'un grand intérêt ainsi que la disposition des chapitres de l'ouvrage que Ežov intitula dans sa dernière version Ot frakcionnosti $k$ fašizmu (De la fraction au fascisme).

2. RCHIDNI, f. 17, op. 3, d. 1004,1. 11. 
Tout en assumant la direction des cadres du parti et des soviets, ainsi que des cadres économiques et militaires du pays, Ežov accumulait sur eux des renseignements compromettants - ces documents ont été répartis en différents dossiers et classés par nom de famille. Les listes des années 1934 à 1936 comportant de brefs jugements sur les opposants de Moscou et le fichier les concernant (31 dossiers) présentent un grand intérêt. Il convient de remarquer que les archives disposaient déjà de documents similaires. Au RCHIDNI ils sont conservés avec le fonds du Comité Central dans une collection à part, en tant que documents sur les fractions au sein du parti de 1921 à 1937 (f. 17, op. 71). Le recensement des cadres du parti était mené au Comité Central par l'ORPO que couvrait Ežov dans les années 1930 en tant que secrétaire du CC. Etant donné que les documents des sections du Comité Central n'ont pas été conservés pour cette période, le fonds Ežov complète de façon substantielle les sources pour la période de la « Grande Terreur ».

L'activité de Ežov au NKVD est représentée sous forme de ses notes personnelles destinées à Stalin, de blocs-notes portant de brèves notations sur les tâches à accomplir ou les conversations, ainsi que de listes de noms de personnes arrêtées avec des indications sur les arrestations et le déroulement des interrogatoires. Une série de lettres importantes de Ežov à Stalin concerne les circonstances de sa destitution du poste de commissaire aux Affaires intérieures. Le fonds renferme des informations sur la nature des documents conservés au secrétariat du NKVD. Ainsi, à partir des documents reçus des secrétariats de Ežov au NKVD et au Comité Central, et indépendamment du fonds propre de Ežov, ont été formés des fonds personnels particuliers, tels ceux de A. S. Enukidze, L. D. Trockij, G. Ja. Sokol'nikov, G. V. Čičerin, et une part importante des fonds de L. B. Kamenev, G. E. Zinov'ev et d'autres.

Le fonds A. Ja. Vyšinskij témoigne de l'éparpillement des archives accumulées lors de l'activité des dirigeants soviétiques. Après la mort de Vyšinskij, le Présidium du Comité Central nomma, le 23 novembre 1954, une commission composée de P. N. Pospelov, secrétaire du CC, de Savčenko, premier vice-ministre du ministre des Affaires étrangères, A. A. Gromyko ; conformément à la mission qui lui était confiée, elle prit connaissance des archives de travail de Vyšinskij, conservées au ministère des Affaires étrangères, au Conseil des ministres, au Ministère public, à la Cour Suprême, au ministère de la Justice, à l'Académie des sciences, à son appartement, à sa résidence secondaire et à son cabinet. Comme il s'agissait surtout de correspondance professionnelle, la commission décida de ne pas créer de fonds spécial dans le CPA.

Par décision du Comité Central, «Sur l'activité professionnelle et les archives personnelles de A. Ja. Vyšinskij », il fut décidé de conserver les documents dans les archives des départements correspondants ${ }^{3}$. En conséquence les archives de Vyšinskij sont aujourd'hui localisées : 1) au ministère des Affaires étrangères où sont conservés les papiers du secrétariat de Vyšinskij pour les années 1940-1953 se rapportant à l'activité du ministère des Affaires étrangères, y compris les notes

3. RCHIDNI, f. 588, op. 2, d. 154,11. 1-3. 
personnelles sur le travail du ministère ; 2) au Conseil des Ministres de l'URSS : des documents reflétant l'activité de Vyšinskij au poste de vice-président du SNK, il s'agit pour l'essentiel de la correspondance à propos de l'organisation et du financement des institutions scientifiques et d'enseignement ; 3) au Ministère public, au ministère de la Justice et à la Cour Suprême sont conservés des documents sur les interventions de Vyšinskij aux procès de la fin des années 1920 et du début des années 1930, sur son rôle dans l'élaboration des circulaires concernant les enquêtes des procès politiques et criminels ainsi que la pratique judiciaire, les sténogrammes de discours et de réquisitoires, la correspondance avec le Comité Central, le SNK, la KPK, le NKVD, portant sur les projets de résolutions de justice et les sentences prononcées, sur les actes de violation de la législation socialiste, les dénonciations au Comité Central du parti et au SNK d'organisations et de groupes «contrerévolutionnaires », sur la propagande antisoviétique, les actes de sabotage, etc ; des décrets, des directives et des circulaires des années 1931 à 1933 rédigées ou signées par Vyšinskij. Il n'est donc pas étonnant que le fonds Vyšinskij entré au RCHIDNI en provenance des Archives du Président n'ait été constitué que de trois dossiers (en 1955, le ministère des Affaires étrangères transféra ces dossiers aux Archives du Politbjuro car il estimait qu'ils ne correspondaient pas à son profil. Ce sont : le dossier 1 - documents d'information de la commission pour la réception des archives de Vyšinskij ; les dossiers 2 et 3 - originaux et copies de documents des années 1935 à 1940 dont le texte de l'introduction du verdict d'accusation dans le procès du bloc antisoviétique trotskiste de droite, le canevas d'une partie du réquisitoire du procureur et le texte de la déclaration destinée à la presse, le tout écrit par Stalin en février 1938, le projet de la décision du Ministère Public, du Commissariat du peuple à la Justice et de la Cour Suprême d'URSS (1940) sur la bonne application du décret de la Cour Suprême du 26 juin 1940 concernant les femmes ayant des enfants non sevrés et en bas âge et portant également la décision de Stalin ainsi que d'autres documents.

Le fonds de G. K. Ordžonikidze fut constitué selon un schéma analogue. En 1993, des documents supplémentaires d'Ordžonikidze provenant des Archives du Président sont arrivés au RCHIDNI, en tout 4 dossiers : le dossier 1 concerne les lettres d'Ordžonikidze à A. I. Rykov, L. I. Mirzojan, G. L. Pjatakov (en tout 15 folios, dont 12 comportent au verso l'inscription au crayon « en provenance des archives de Ežov »; le dossier 2 - des documents du Département de la police conservés par Ordžonikidze dans deux enveloppes cachetées de cinq cachets de cire avec l'inscription «Ne pas décacheter en mon absence » (une enveloppe fut décachetée le 28 octobre 1937 par Mikojan et Berija, comme en témoigne un émargement de Mikojan, cf. 1. 30 du dossier) le dossier 3 contient les inventaires des documents rédigés en 1937 par la commission du Comité Central pour la réception des archives d'Ordžonikidze, et le dossier 4 contient les actes du transfert des archives d'Ordžonikidze à l'Institut du marxisme-léninisme (IML) en 1946.

La confrontation entre d'une part les inventaires rédigés par la commission pour la réception des archives d'Ordžonikidze et les actes du transfert des documents à l'IML et à d'autres institutions, et d'autre part les documents de contrôle du fonds 
$n^{\circ} 85$ conservés au RCHIDNI a montré que presque toutes les archives avaient été réparties par suite d'une décision de la commission. Le 29 octobre 1937, la commission (Mikojan, Mehlis, Berija, Semuškin) informa Stalin qu'elle avait achevé son travail sur la réception des archives d'Ordžonikidze au Commissariat à l'industrie lourde et à son appartement. De la lettre de la commission, il ressort que les archives furent divisées en plusieurs parties :

- la correspondance de Lenin avec Ordžonikidze et les télégrammes d'Ordžonikidze adressés simultanément à Lenin et à Stalin ;

- la correspondance d'Ordžonikidze avec Stalin, les documents du Politbjuro avec les résolutions et les remarques de Stalin ;

- les lettres des membres et des candidats à devenir membres du Comité Central ;

- les lettres des dirigeants ;

- les lettres d'autres personnes ;

- les lettres et les déclarations de Zinov'ev, Kamenev, Trockij, Pjatakov, V. V. Lominadze, S. I. Syrcov, les documents de la Commission Centrale de Contôle $(\mathrm{CKK})$;

- les documents sur l'activité d'Ordžonikidze dans l'Armée Rouge, en Transcaucasie et en Perse;

- les sténogrammes, les discours, les articles.

(Dans la lettre les noms de LENIN, STALIN, ORDŽONIKIDZE sont écrits en majuscules, alors que les noms de famille des «ennemis du peuple » comme Zinov'ev, Kamenev, Trockij, Radek sont tout en minuscules : zinov'ev, kamenev, etc).

Toutes les archives ont été classées par la commission en 42 inventaires. Il faut dire que les archives de l'appareil d'Ordžonikidze étaient très bien organisées et classées, comme on peut en juger d'après les listes de transmission de dossiers d'un secrétaire à un autre. Il apparaît clairement que, dès 1920, une nomenclature des dossiers était mise en place et que tous les documents adressés au nom d'Ordžonikidze étaient classés dans des cartons. Carton 1 : Moscou (avec, à l'intérieur du carton, un classement par organismes : SNK, ministère du Commerce extérieur (Vneštorg), Conseil militaire révolutionnaire (Revoljucionnyj Voennyj Sovet RVS) ; carton 2 : la Perse ; carton 3 : la Géorgie ; 4 : l'Azerbaïdjan et le Comité Central du PC azéri ; 5: le Comité régional et le SNK de Transcaucasie (Zakkraïkom et ZakSNK) ; 6: l'Arménie ; 8 : la correspondance privée ; 9 : le Comité Central du parti; 10 : l'armée... ; 14 : les plenums du CC ; 15 : le Secrétariat du CC, etc. La nomenclature adoptée en 1920 fut conservée au cours des années suivantes. On se contenta d'ajouter de nouvelles rubriques sous de nouveaux numéros. En principe, la commission n'avait qu'à inventorier les cartons sans les passer au crible.

Dans sa lettre à Stalin, la commission signalait qu'elle avait envoyé en temps opportun une série de documents sur les «traîtres du parti et les ennemis du peuple » à Ežov au NKVD (ce sont les dépositions et les procès-verbaux des interrogatoires de Pjatakov, Radek, A. I. Emšanov, B. Mdivani, N. A. Uglanov, les 
notes d'information spéciales du NKVD, les documents sur les sabotages dans l'industrie, etc).

On envisageait de répartir les documents restants de la façon suivante : la correspondance avec Lenin à l'Institut Marx-Engels-Lenin (IMEL), les documents du Conseil Suprême à l'Économie (Vysšij Sovet Narodnogo Hozjajstva - VSNH) et du Commissariat à l'Industrie lourde (Narodnyj Komissariat Tjaželoj Promyšlennosti - NKTP) au NKTP, ceux portant sur l'activité du PC de Géorgie au Comité Central de Géorgie, sur l'activité du PC d'Arménie et d'Azerbaïdjan aux Comités Centraux respectifs de ces républiques ; les documents du Politbjuro avec les résolutions et les remarques de Stalin, les lettres et les télégrammes de Stalin, des membres du Politbjuro et du Comité Central, les documents de la Commission Centrale de Contrôle aux archives du Politbjuro ${ }^{4}$.

On a conservé des reçus signés par des collaborateurs du NKVD, du NKID et du Secteur Spécial du Comité Central auxquels la commission avait envoyé des documents en 1937.

D'après un dépouillement préliminaire et selon les inventaires établis par la commission, les archives d'Ordžonikidze comptaient près de 16000 folios. Il était prévu de rendre 3814 folios au NKVD, 1433 au NKID, 670 à la direction des renseignements de l'Armée Rouge, 4390 au NKTP, 5384 au Secteur Spécial du Comité Central.

Les matériaux destinés à retourner au NKTP ont été laissés au Secrétariat de Mikojan et ce n'est qu'en 1961 et 1966 qu'ils ont été transmis aux Archives Centrales du Parti par le secrétaire de A. I. Mikojan, Barabanov.

Les documents sélectionnés pour être transmis à l'IMEL ont été conservés jusqu'en 1946 aux archives du Politbjuro et ce n'est qu'après la guerre, sur la demande de l'IMEL et conformément à l'ordre de Poskrebyšev, qu'ils furent rendus. La vérification des inventaires permet de constater que la quasi-totalité des documents destinés à l'IMEL lui ont bien été transmis, à l'exception de la correspondance avec Stalin (695 folios d'après l'inventaire de la commission du Comité Central). Une note de Rezakova, employée aux Archives Centrales du Parti, chargée de la réception et de l'examen du fonds, témoigne du fait qu'une partie des lettres de Stalin est restée aux archives du Politbjuro. En août 1947, elle écrit : «Sur la décision du président de la commission du CC pour la réception des archives personnelles d'Ordžonikidze appartenant à A. I. Mikojan, le groupe de lettres de Stalin à Ordžonikidze des années 1923 à 1932 a été laissé au Secteur Spécial du Comité Central [...] On ne peut pas dire que le fonds personnel soit complet $»^{5}$.

Parmi les fonds nouvellement reçus des Archives du Président, ceux de Molotov, Kaganovič et Vorošilov sont les plus intéressants pour les chercheurs parce qu'ils sont complets.

Les documents constituant le fonds personnel de Molotov - 1669 dossiers comprennent des documents originaux et des copies avec des annotations de

4. RCHIDNI, delo fonda N. 85, t. 1, č. 5, 11. 154-156.

5. Ibid.,1. 1. 
Molotov et couvrent la période 1907 à 1986. Le fonds a été rassemblé dans les archives du Comité Central à partir de plusieurs sources : le Conseil des Ministres, le CC du PCUS mais aussi des documents envoyés par la famille de Molotov après sa mort et provenant de son appartement et de sa datcha.

Les dossiers ont été classés pour l'essentiel par thèmes et reflètent les différentes étapes de l'activité de Molotov : son activité avant la révolution, au CC du POSDR et au comité de Petrograd, en qualité d'envoyé du CC du PC(b)R dans la région de la Volga, de secrétaire du CC, de membre du Politbjuro du CC et du Comité Exécutif Central ; en qualité de secrétaire du Comité du Parti à Moscou, au Comité Exécutif de l'Internationale Communiste (Ispolnitel'nyj Komitet Kommunističeskogo Internacionala - IKKI), président et vice-président du Conseil des Commissaires du peuple et du Conseil du travail et de la défense (Sovet Truda i Oborony - STO), du Conseil des ministres, en qualité de commissaire du peuple et ministre des Affaires étrangères d'URSS, etc.

Les documents reflétant le travail de Molotov comme membre du Comité Central du parti sont particulièrement intéressants. Ils permettent de compléter de façon substantielle les sources de l'histoire du parti et de l'État soviétique. Cela concerne surtout l'histoire de la société soviétique de la fin des années 1920-1930. Comme les archives de l'appareil du CC concernant les années 30 ont été mal conservées, les documents de ce fonds deviennent une source importante pour le fonctionnement des instances supérieures du parti. Ainsi le fonds contient une grande quantité de documents envoyés à Molotov en tant que membre du Politbjuro et qui concernent des questions internes au parti. Par exemple, l'histoire de la publication et de la critique de l'article de Buharin «Ekonomika sovetskoj strany » (L'économie du pays soviétique) dans les Izvestija (en 1934), les déclarations de G. E. Zinov'ev, A. N. Slepkov, D. P. Mareckij, A. G. Ÿljapnikov après leur arrestation, les notes du NKVD sur ces affaires, etc.

Molotov, tout comme Stalin, a constitué lui-même ses archives ainsi qu'en témoigne l'inscription de Molotov « Mes archives » sur de nombreux documents. Des documents analogues se trouvent dans les fonds de Kaganovič, Andreev, Mikojan, Vorošilov et d'autres.

L'étude des documents contenus dans les fonds nouvellement arrivés des Archives du Président permet de tirer les conclusions suivantes :

D'abord, le dépouillement scientifique des documents de Buharin, Rykov, Ordžonikidze, Jaroslavskij n'a pas été effectué. Les inventaires sont en fait une énumération de dossiers sans datation ni précisions quant à l'auteur et au destinataire, et sans description des documents, comme il est d'usage dans les archives. Les documents sont assemblés dans des classeurs, le texte est souvent troué par des poinçons, ce qui conduit inévitablement à la perte de fragments de manuscrit. La description des dossiers a un caractère tellement général qu'une part importante des documents contenus dans ces dossiers en est exclue.

Ensuite, une étude plus attentive des inventaires des fonds qui, en apparence, correspondent aux normes habituelles de dépouillement des documents, permet de constater qu'aucun travail (épigraphique et autre) ne fut fait sur ces dossiers 
complexes; le plus souvent l'atribution et la date du document sont erronées et différents folios d'une même lettre se retrouvent parfois dans des dossiers différents.

Enfin, lors du transfert des documents des Archives du Président, la règle principale de l'archivistique a été transgressée : le principe de l'indivisibilité du fonds selon lequel chaque fonds doit être transféré dans sa totalité et avec tous les documents concernant son inventaire, y compris l'historique de sa constitution et de sa formation, où sont mentionnés toutes les arrivées et tous les transferts de documents.

Tout cela ne permet pas de répondre à la question essentielle que se posent l'archiviste et l'utilisateur : tous les documents sont-ils connus à l'heure actuelle ou bien est-ce que, comme par le passé, une certaine partie d'entre eux reste encore en dehors des fonds accessibles. Cette question n'est pas fortuite. Au moment de la transmission du fonds Molotov au RCHIDNI, une partie des documents inclus dans l'inventaire (24 dossiers) a été déclassée à la dernière étape du transfert, ce dont témoigne une note dans l'inventaire « dossier déclassé le 12 octobre 1994 ». Dans l'inventaire seul le numéro du dossier est spécifié sans autre indication. Manque également la mention du nouvel emplacement de ces documents : sont-ils restés aux Archives du Président ou ont-ils été transmis, par exemple, au ministère des Affaires étrangères.

Ainsi on peut malheureusement dire que les fonds personnels ont été constitués de façon assez désordonnée, sur la base de décisions fortuites. En général, ces fonds ont été fractionnés et redistribués entre les différents départements et ministères, ce qui entrave le travail des chercheurs qui doivent en tenir compte.

(Traduit du russe par Nicolas Spassky)

Rossijskij Centr Hranenija i Izučenija Dokumentov Novejšej Istorii

103821 Moscou

ul. Bol'šaja Dmitrovka, 15 